\title{
The Hippo pathway transcriptional co-activator YAP is involved in head regeneration and bud development in Hydra
}

1 Manu Unni ${ }^{1}$, Puli Chandramouli Reddy ${ }^{1}$ and Sanjeev Galande *1

$2{ }^{1}$ Centre of Excellence in Epigenetics, Department of Biology, Indian Institute of Science Education

3 and Research, Pune, India

4 * Correspondence:

5 Corresponding Author

6 sanjeev@iiserpune.ac.in

7 Keywords: Hydra, Hippo Pathway, YAP, Regeneration.

8 Abstract

9 The Hippo signaling pathway has been shown to be involved in the regulation of cellular identity,

10 cell/tissue size maintenance and mechanotransduction. The Hippo pathway consists of a kinase

11 cascade which determines the nucleo-cytoplasmic localization of YAP in the cell. YAP is the effector 12 protein in the Hippo pathway which acts as a transcriptional cofactor for TEAD. Phosphorylation of

13 YAP upon activation of the Hippo pathway prevents it from entering the nucleus and hence abrogates

14 its function in transcription of target genes. In Cnidaria, the information on the regulatory roles of the

15 Hippo pathway is virtually lacking. Here, we report for the first time the existence of a complete set

16 of Hippo pathway core components in Hydra. By studying their phylogeny and domain organization,

17 we report evolutionary conservation of the components of the Hippo pathway. Protein modelling

18 suggested conservation of YAP-TEAD interaction in Hydra. We also characterized the expression

19 pattern of the homologs of yap, hippo, mob and sav in Hydra using whole mount RNA in situ

20 hybridization and report their possible role in stem cell maintenance. Immunofluorescence assay

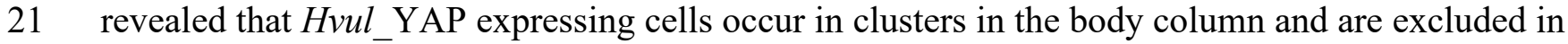

22 the terminally differentiated regions. The YAP expressing cells are recruited early during head

23 regeneration and budding implicating the Hippo pathway in early response to injury or establishment

24 of oral fate. These cells exhibit a non-clustered existence at the site of regeneration and budding,

25 indicating the involvement of a new population of YAP expressing cells during oral fate

26 specification. Collectively, we posit that the Hippo pathway is an important signaling system in 
27 Hydra, its components are ubiquitously expressed in the Hydra body column, and may play crucial

28 role in Hydra oral fate specification.

\section{Introduction}

30 The ability of the cells to come together and act in a coordinated fashion helped organisms evolve from solitary single-cell based forms to extremely complex and specialized multicellular organisms. Signaling pathways which allow cell-cell communication in a highly specific and spatio-temporal manner enabled the advent of multicellularity. This is evident while studying ontogenesis.

34 Considering the sheer number of cell-types present in a complex organism such as humans, it comes as a surprise that the development of an organism from a zygote to a fully formed adult is controlled by a complex interplay of merely 10 main classes of signaling pathways (Perrimon et al., 2012). These include- Notch, Wnt, Hedgehog, TGFß/BMP, Receptor-tyrosine kinase (RTK), Hippo, NF$\boldsymbol{\kappa}$ B, JAK-STAT, JNK \& Nuclear receptor signaling pathway family.

Multicellularity arose about 400-1000 million years ago on earth (Butterfield, 2000) independently in at least 16 different eukaryotic lineages which led to complex multicellular taxa like metazoa, Fungi and Embryophyta (Butterfield, 2000;Brunet and King, 2017). Considering the bilaterians as the most complex and diverse multicellular clade, a basic 'Developmental Toolkit' required for generation, organization and maintenance of multicellular structures can be assessed. The origin of these developmental tools which include transcription factors, signaling pathways, cell adhesion and cell polarity related genes can be traced back to basal metazoans (Tweedt and Erwin, 2015). A detailed analysis of these development toolkits, body plan and differential germ layers and a diverse cell-type system indicates Cnidarians are arguably the first phylum to evolve and exhibit features which underlie the traits commonly seen in Bilateria. Cnidarians exhibit an oral-aboral body axis polarity with a diploblastic germ layer organization. These germ layers in cnidarians have been reported to form myoepithelial cells, nerve-net of sensory/ganglion neuronal cells, gastric cells, germline cells and cnidocytes which are the defining feature of the phylum. Studies in the past few decades have shown clearly that these primitive organisms display highly complex developmental programs and toolkits which are commonly found in the bilaterians.

Among the cnidarians, Hydra is the best-characterized model. Hydra is a freshwater polyp known to exhibit tremendous regenerating potential with a capability to regenerate even from reaggregated cells of dissociated polyps (Gierer et al., 1972). It has been a classical model for developmental and 
regeneration biology for more than two centuries and has contributed immensely towards the understanding of morphogen mediated processes and understanding various cell signaling pathways (Reddy et al., 2019b). Among the ten developmentally important signaling pathways as discussed above, seven of them have been shown to be functional according to the studies on Hydra. Many components of the Wnt signaling have been reported in Hydra and their role has been established to be important in the regulation of head organizer activity (Hobmayer et al., 2000). Many components of Notch signaling are present in Hydra and have been reported to be important in the boundary formation in tissues (Sprinzak et al., 2010; Münder et al., 2013). The TGF $\beta$ superfamily of signaling pathway has also been reported to be crucial for Hydra developmental signaling such as during tentacle formation, foot formation, symmetry breaking (Reinhardt et al., 2004; Rentzsch et al., 2007; Watanabe et al., 2014). Members of the RTK family of signaling pathways - VEGF, FGF and Ephrin have been shown to be crucial for regeneration in Hydra (Tischer et al., 2013; Krishnapati and Ghaskadbi, 2014). NF- $\boldsymbol{\kappa}$ B has been reported to be important for early regenerative time points in Hydra (Franzenburg et al., 2012; Wenger et al., 2014). While their role is presently thought to be innate immunity/inflammation-related, its direct developmental regulation is yet to be established. JNK in Hydra has been found to be crucial in nematocyte differentiation and regulation of TLRsignaling (Philipp et al., 2005; Franzenburg et al., 2012). Among the nuclear receptor family of signaling pathways, Retinoblastoma gene has been found to be expressed in almost all cell types in Hydra but its specific role has not been deciphered (Schenkelaars et al., 2018). Another nuclear receptor protein, NR3E has been found to be expressed in Hydra and is predicted to respond to the parasterol A, a cnidarian A-ring aromatic steroid (Khalturin et al., 2018). Among the three remaining developmentally important signaling pathways yet to be reported in Hydra are- Hedgehog, JAKSTAT and Hippo signaling.

Hippo pathway has emerged as a major player for the orchestration of spatio-temporal regulation of cell differentiation, proliferation, tissue size control, and apoptosis. These capabilities enable the Hippo pathway to be important in the regulation of morphogenesis and tissue or organ regeneration. It was first described and reported in Drosophila while screening for tumour suppressor genes in 1995 (Xu et al., 1995). However, it was only in 2005 when Yorkie (Yki), a transcription co-activator, 
highly conserved mammalian homolog of the Drosophila Yki. The Hippo core components are

kinases which phosphorylates YAP through a cascade, which represses its transcriptional activity by preventing its nuclear transportation and hence its interaction with transcription factors like TEAD

(Fulford et al., 2018). Upon phosphorylation, YAP is sequestered in the cytoplasm through 14-3-3 interaction or undergo ubiquitination for its degradation. The core components of Hippo characterized in Drosophila consists of Ser/Thr kinases- Hippo (Hpo) and Warts (Wts); and their adapter proteins- Salvador (Sav) and Mats. In mammalians, the equivalent set of factors is named asMst, Lats, Sav and Mob, respectively. At the cellular and molecular levels, the Hippo pathway and its functions are highly conserved between invertebrates and vertebrates.

There has been a paucity of literature to date about Hippo signaling in basal metazoans. The role of Hippo signaling in highly regenerative organisms like Hydra is unknown. A recent study in another cnidarian reported that Clytia hemispherica has all the core components of Hippo pathway and CheYki has cell proliferation regulatory function. Hence, it is pertinent to characterize the homologs of core Hippo pathway components in Hydra to understand their role in Hydra regeneration and cell proliferation and differentiation. A recent study has reported the presence of core components of the Hippo pathway in Nematostella (Hilman and Gat, 2011), suggesting that this pathway has fairly conserved ancient origin during the evolution of multicellular organisms. Here, using a combination of bioinformatic analysis and molecular cloning, we report the existence of a complete set of core Hippo pathway components in Hydra. Using domain analysis and 3D protein modelling, we show that these homologs have a conserved domain and motif architecture indicating a possible conserved interactive signaling network. Whole-mount in situ hybridization (WISH) analysis revealed that these genes are expressed across the body column with a few gene-specific variations. Adapting CheYki specific antibody for immunofluorescence assay of Hydra YAP, we show that nuclear localized YAP

111 occurs as clustered cells across the body column with no expression in the regions which are

112 terminally differentiated. We show that the YAP expressing cells are recruited to the regenerating tip

113 and early buds. Further, we report the existence of a separate non-clustered nuclear localized YAP

114 expressing cell population at the hypostomal region which may be involved in oral fate specification 115 and maintenance. 


\section{Materials and Methods}

\section{Animal culture}

121 Clonal culture of Hydra vulgaris Ind-Pune (Reddy et al., 2011) was maintained in Hydra medium by

122 following standard methods at $18 \pm 1^{\circ} \mathrm{C}$ (Horibata et al., 2004). Polyps were fed daily with freshly

123 hatched Artemia nauplii larvae and washed 6-8 h after feeding. For regeneration experiment, Hydra

124 polyps starved for $24 \mathrm{hrs}$ were decapitated just below the tentacle base and allowed to regenerate till

$1250,1,2,4$ and 8 hours post amputation (hpa). The polyps were then fixed and processed for

126 immunofluorescence assay. For budding experiment, Hydra polyps starved for 24 hrs were collected

127 at different stages of bud development. These were then fixed and processed for immunofluorescence

128 assay. The different stages of budding were identified and labelled as reported previously (Otto and

129 Campbell, 1977).

\section{Identification of Hippo pathway homologs in Hydra}

132 Hydra magnipapillata genome draft comprising $82.5 \%$ of 1.05 Gbp sequenced genome available as

133 Refseq was initially used for identifying Hippo Pathway core components (Chapman et al., 2010).

134 This assembly turned out to be incomplete and we were unable to fish out any homologs. An in-

135 house transcriptome assembly generated in the Galande laboratory (Reddy et al., 2019a) was

136 therefore used for the present study. To further improve the assembly, the in-house transcriptome was

137 merged with the NCBI RefSeq to generate a hybrid assembly. The hybrid assembly was found to be

$13899.6 \%$ complete as compared to $95.7 \%$ exhibited by NCBI RefSeq (Reddy et al., 2019a). Using the

139 stand-alone NCBI BLAST program, hits of homologs of Hippo pathway core components were

140 identified (Madden, 2013). To confirm the hits, Reverse BLAST was performed. Finding a hit of a

141 homolog in different phyla or species would confirm the homolog status. To further confirm, the

142 amino acid sequences of these homologs were searched in HMMER (Hmmer, RRID:SCR_005305)

143 for affirmation based on the hits returned (Potter et al., 2018). Once the homologs were identified,

144 further analyzed for domain organization by SMART (SMART, RRID:SCR_005026) (Letunic et al., 145 2002). After manual evaluation of the domain organization, the domain architecture was constructed 146 to scale using DOG 2.0 software (Ren et al., 2009). 


\section{Molecular phylogenetic trees}

149 Sequences from different representative phyla were collected based on protein BLAST searches using

150 Human YAP sequence as query. The collected sequences were aligned using MUSCLE (Edgar, 2004).

151 The alignment was trimmed using automated trimAl programme (Capella-Gutiérrez et al., 2009). This

152 alignment was subjected for phylogenetic analysis using FastTree 2 to generate an approximately

153 maximum likelihood (ML) tree (Price et al., 2010). This method was selected after testing PhyML and

154 RaxML as FastTree 2 has given better confidence on branching points and this could be due to highly

155 divergence nature of the sequences. This pipeline was implemented in online platform NGphylogeny.fr

156 (Lemoine et al., 2018). Here, LG substitution model was used with Felsenstein's phylogenetic

157 bootstrap with a value of 1000 (Lemoine et al., 2019). Phylogenetic tree was visualized by using iTOL

158 webserver (Letunic and Bork, 2019). Tree was rooted using Amphimedon queenslandica YAP-like

159 sequence as an outgroup. The domain organization analysis and visualization were carried out using

160 DoMosaics software (Moore et al., 2014) using embedded HMMER3 tools (Mistry et al., 2013) and

161 Pfam data. The sequences details were provided in Supplementary Table 1.

162 For the analysis of the rest of the Hippo pathway components, alignments and molecular

163 phylogenetic trees of the protein sequences were carried out using MEGA 6.0 software (Tamura et

164 al., 2013). MUSCLE algorithm was used for amino acid sequence alignment (Edgar, 2004). The

165 alignment was graphically represented using Jalview (Waterhouse et al., 2009).

\section{Cloning of Hippo pathway homologs from Hydra}

168 Total RNA was extracted from Hydra polyps starved for $48 \mathrm{hrs}$ and cDNA was synthesized from

169 total RNA using Improm-II reverse transcriptase system (Promega ${ }^{\mathrm{TM}}$ ) according to the

170 manufacturer's instructions. Hippo pathway genes were amplified by polymerase chain reaction

171 using Pfu DNA polymerase with the following primers:

172 Hvul_yap_forward:5'ATGGATATGAATTCTACGCAACGGC3',

173 reverse: 5'CTACAACCAAGTCATATATGCATTAGGC3';

174 Hvul_tead_forward:5'ATGGCGGAAAACTGTCGAGATCC3',

175 reverse: 5'TCAGTCTCTGACTAATTTAAATATGTGGT3'; 
Hvul_hpo_forward:5'ATGTCTCGCAGTTTGAAGAAGTTGAG3',

177 reverse: 5'TTAAAAATTTGCTTGCCTGCGTT3';

178 Hvul_mob_forward:5'ATGAGTTTCCTGTTTGGCTCCA3',

179 reverse: 5'TTATTTATTAATTAACTTATCCATAAGTTC3';

Hvul_lats_forward:5'ATGGCAGCTAATAATCTTTTTAGTAG3',

reverse: 5'TCATACAAAAACAGGCAACTTGC3';

Hvul_sav_forward:5'ATGTTTAAGAAAAAAGATATTATCAAAACA3',

184 The PCR conditions: Initial denaturation at $94^{\circ} \mathrm{C}$ for $5 \mathrm{~min}$, followed by 30 cycles of denaturation at

$18594^{\circ} \mathrm{C}$ for $30 \mathrm{sec}$, annealing at the respective annealing temperatures (Ta) for $45 \mathrm{sec}$ and extension at

$18672^{\circ} \mathrm{C}$ for $45 \mathrm{sec}$ with the final extension $72^{\circ} \mathrm{C}$ for 5 minutes. The PCR amplified products were gel

187 eluted using Mini elute kit (Qiagen), followed by A-tailing reaction using KapaTaq enzyme and

188 cloned in pGemT-Easy vector system (Promega ${ }^{\mathrm{TM}}$ ) or TOPO TA cloning vector as per the

189 manufacturer's instructions. The recombinant plasmids were sequenced using sequencing primers

190 and the nucleotide sequences of cloned genes were deposited at NCBI Genbank (Hvul_yap-

191 MW650883; Hvul_tead-MW650884; Hvul_hpo-MW650879; Hvul_mob-MW650880; Hvul_lats-

192 MW650881 and Hvul_sav-MW650882).

\section{Whole mount in situ hybridization}

194 Digoxigenin-labelled sense and antisense RNA probes were prepared by in vitro transcriptions using recombinant plasmids of target genes made as mentioned above (Roche Life Science) and used for in

196 situ hybridization. Whole mount in situ hybridization was performed on the polyps as described by

197 Martinez et. al., (1997) with the following changes (Martinez et al., 1997). The animals were relaxed

198 for $2 \mathrm{~min}$ in $2 \%$ urethane. Treatment with proteinase-K was performed for an optimum of 15 min and

199 heat-inactivation of the endogenous alkaline phosphatases was done at $70^{\circ} \mathrm{C}$ for 15 min in $1 \mathrm{XSSC}$.

200 Digoxigenin labelled RNA probes at a concentration of 200-600 ng/ml of the probe was used for

201 hybridization at $59^{\circ} \mathrm{C}$. The post-hybridization washes were performed using $1 \mathrm{X}$ SSC-HS gradients.

202 After staining with BM-purple AP substrate for $30 \mathrm{~min}-1 \mathrm{hr}$ at room temperature, the animals were 
mounted in $80 \%$ glycerol for imaging. Imaging was carried out using $10 \mathrm{X}$ DIC objective lens with

Axio Imager Z1 (Zeiss).

\section{Cryosectioning of WISH stained Hydra samples}

206

207

208

209

210

211

212

213

214

215

216

217

218

219

220

221

222

223

224

225

226

227

228

229

230

The stained polyps were rehydrated to PBS gradually through PBS: methanol gradient $(25 \%, 50 \%$, $75 \%$, and $100 \%$ wash each for 10 mins). These polyps were then shifted to a $30 \%$ sucrose solution by gradually taking it through $10 \%$ and $20 \%$ for 30 mins each. The polyps were left in $30 \%$ sucrose overnight. These polyps were then embedded in $10 \%$ PVP (polyvinyl pyrrolidone) by making cubes of PVP $\left(1 \times 1 \times 2 \mathrm{~cm}^{3}\right)$ made from aluminum foil cast. The embedded polyps were then sectioned ( 25 $\mu \mathrm{m}$ thick) using Leica CM1950 - Cryostat. The sectioned ribbons were then collected on a glass slide and covered and sealed under a coverslip. The sectioned were then photographed under ZEISS Axio Zoom V16 apotome microscope.

\section{Analysis of expression of Hippo pathway components from single-cell transcriptome profile}

t-SNE plots and gene expression plots of Hippo pathway components were generated and extracted from the Single Cell Portal (https://portals.broadinstitute.org/single cell/study/SCP260/stem-celldifferentiation-trajectories-in-Hydra-resolved-at-single-cell-resolution). In order to use the Single Cell Portal, gene IDs of Hippo pathway components were acquired through a BLAST search in the Juliano aepLRv2 nucleotide database via Hydra 2.0 Genome Project Portal (https://research.nhgri.nih.gov/Hydra/sequenceserver/). To determine the clusters of cells that express individual Hippo pathway components, differential gene expression was analyzed using edgeR, which is a tool to analyze RNA-seq data using the trimmed mean of M-values (TMM) method. Differential gene expression was calculated as fold change.

\section{Immunofluorescence staining}

A recently published paper reported the presence of Yorkie (YAP/Yki) in Clytia hemispherica and producing polyclonal antibody specific to CheYki in rabbit against the peptide FNRRTTWDDPRKAHS (Coste et al., 2016). This antibody along with the pre-immune serum was kindly gifted by Dr Michaël Manuel (Sorbonne Universités, Université Pierre et Marie Curie (UPMC), Institut de Biologie Paris-Seine (IBPS) CNRS). The antibody was validated by immunofluorescence analysis. 
231 Immunofluorescence assay was performed as per the protocol is given in Takaku et. al., 2014

232 (Takaku et al., 2014). Hydra polyps were starved at least for one day before fixation. Animals were

233 relaxed in 2\% urethane for 1-2 min and fixed in 4\% paraformaldehyde (in 1XPBS) overnight at $4{ }^{\circ} \mathrm{C}$

234 or $1 \mathrm{hr}$ at RT. 1:100 concentration of primary antibody was used. 1:100 concentration of Invitrogen

235 Alexa-conjugated secondary antibodies was used. Invitrogen Alexa 488 conjugated Phalloidin for

236 staining. DAPI was used for nuclear staining. These samples were then imaged on ZEISS Axio Zoom

237 V16 (for regeneration and whole animal images) or Andor Dragonfly Spinning Disc (for budding

238 Hydra) microscopes.

239 Modelling

240 The $2.8 \mathrm{~A}^{\circ}$ crystal structure of the human YAP-TEAD complex deposited on PDB (4RE1) was as a

241 reference for modelling the TEAD binding domain and YAP binding domains of Hydra YAP-TEAD

242 complex (Zhou et al., 2015). Modeller software (Webb and Sali, 2016) was used to build five

243 optimum models based on 4RE1 in the multi-model mode. Among the five models, the model with

244 minimum DOPE assessment score and maximum GA341 assessment score was chosen for the final

245 analysis. The model was then visualized in CHIMERA for analysis, superpositioning and annotation

246 (Pettersen et al., 2004). The non-covalent bond analysis was performed using Biovia Discovery

247 Studio Visualizer (BIOVIA, 2017). The Binding energy calculations were performed using

248 PRODIGY web server (Xue et al., 2016).

\section{Results}

251 Characterization and phylogenetic analysis of Hydra Hippo pathway genes

252 Core Hippo pathway homologs - hippo/mst, mob, lats, sav, yap and tead were identified from the in253 house Hydra transcriptome using NCBI stand-alone BLAST (Reddy et al., 2019a). In mammals, 254 hippo, mob, lats and yap have 2 paralogs each while tead has 4 paralogs. Sav, on the other hand, has 255 no reported paralogs. The occurrence of these paralogs has been attributed to whole-genome 256 duplication events correlated to certain fish species (Chen et al., 2019). Therefore, any species 257 evolved earlier than fishes do not contain paralogs as reported for Hippo pathway genes. Conforming 258 to these reports, Hydra consists of only one gene coding for each of the core Hippo pathway 259 components. The Hydra Hippo pathway homologs were labelled as-Hvul_hpo, Hvul_mob, 
Hvul_lats, Hvul_sav, Hvul_yap and Hvul_tead. The presence of these homologs in Hydra was confirmed by obtaining the corresponding amplicons from Hydra cDNA (Supplementary Figure 1). Upon determining the nucleotide percent identity with other reported model organisms used for studying the Hippo pathway, we find that Hydra had a higher percent identity with humans than Drosophila (Figure 1 B). Hvul_hpo shows about $60 \%$ identity with humans and $56 \%$ identity with Drosophila.Hvul_sav is comparatively less conserved with a $24 \%$ identity with human and $22 \%$ identity with Drosophila. Hvul_mob is highly conserved across the animal phyla with about $84 \%$ identity with humans and $83 \%$ identity with Drosophila. Hvul_lats shares $41.6 \%$ identity with humans and $42 \%$ identity with Drosophila. Hvul_yap shows $34.6 \%$ identity with humans and $34 \%$ identity with Drosophila. Hvul_tead exhibits about $65 \%$ identity with humans and $59 \%$ identity with Drosophila.

An earlier study performed the phylogenetic analysis of YAP homologues found in selective phyla (Hilman and Gat, 2011). However, this analysis did not cover majority of invertebrate phyla such as Annelida, Mollusca and Echinodermata. This could be due to lack of reliable data for the identification of the YAP homologues. Here, we have combined the phylogenetic analysis with predicted domain architecture. We used a YAP-like sequence found in Amphimedon queenslandica as an outgroup for rooting the tree. Additionally, a protein sequence with BLAST similarity from a unicellular Eukaryote (Capsaspora owczarzaki) was used for domain organization comparison. In this analysis, we observed that Hydra homologue of YAP exhibits a strong affinity to the chordate counterparts rather than nonchordate homologues (Figure 1C). An interesting observation after inclusion of multiple invertebrate phyla in the analysis is that they are highly diverged compared to the Cnidarian and Chordata species. This can be interpreted based on the weak branch support values (Figure 1C). Additionally, a molluscan homologue, Sepia pharaonic (SEPPH_YAP) showed more similarity with Drosophila Yki and homologues (Figure 1C). Domain organization analysis has led to identification of variability in the Nterminal homology domain (FAM181). This region contains TEAD binding domain (TBD). (Chordata) the FAM181 domain could not be detected (Figure 1C). This could be due to the higher sequence divergence in this region. 
a fully functional pathway consisting of these core components (Figure $1 \mathrm{~A}$ ). Hvul_HPO domain analysis revealed conserved N-terminal Protein kinase domain (PKinase Domain) and a C-terminal SARAH (Salvador-RASSF-Hippo) domain. The presence of these domains indicates the conserved regulation of activation of $\mathrm{Hvul}$ _HPO kinase activity (Glantschnig et al., 2002;Praskova et al., 2004;Boggiano et al., 2011). The Hvul_SAV also can be seen to have conserved the SARAH domain required for orchestrating the reported scaffolding activity (Yin et al., 2013). Hvul_LATS domain architecture indicates conservation of the hydrophobic motif (Motif: AFYEFTFRHFFDDGG) (a 40\% hydrophobicity confirmed using web-based peptide analysis tool at www.peptide2.com/N_peptide_hydrophobicity_hydrophilicity.php) containing the Threonine residue (T993) required for the activation of LATS by HIPPO phosphorylation (T1079 in humans) (Supplementary Figure 2A) (Hergovich et al., 2006;Ni et al., 2015). The MOB binding motif is highly conserved in Hvul_LATS as compared to the human and mouse (Figure 2.4 B). The autoactivation T-loop (near S909 in human) of Hvul_LATS is $100 \%$ conserved (Motif: AHSLVGTPNYIAPEVL) near S830 (Supplementary Figure 2B) (Ni et al., 2015). Hvul_MOB is highly conserved (84\% identity with human MOB) as compared to any other components of Hippo pathway homologs in Hydra indicating highly conserved function. The same site as reported for Human MOB is also highly conserved in Hvul_MOB at T35 (Motif: LLKHAEATLGSGNLR) (Supplementary Figure 2C). This site is crucial for the release of LATS-MOB complex from the MST-SAV-LATS-MOB complex and further initiation of LATS auto-activation (Ni et al., 2015). Hvul_YAP domain analysis revealed that it had a conserved TEAD-Binding Domain (TBD) and two WW domains. A serine phosphorylation prediction for YAP primary sequence was performed using GPS 2.1 web-based tool (Xue et al., 2010). Based on GPS prediction and manual curation, Hvul_YAP is predicted to have LATS phosphorylation site at S74 (motif: PIHTRARSLPSNIGQ) and S276 (motif: YTAYMNSSVLGRGSS) homologous to the S127 (motif:

315 PQHVRAHSSPASLQL) and S381 (motif: SDPFLNSGTYHSRDES) (Supplementary Figure 2D).

316 Similar to mammals, a phosphodegron motif (DSGLDG) was identified immediately downstream to 317 the S276 site (S381 in humans) which could be phosphorylated by CK1-y at S287 (S388 in humans)

318 of Hvul_YAP (Supplementary Figure 2E) (Zhao et al., 2010). These analyses indicated that the

319 Hippo pathway effector protein YAP is well equipped for regulation by the LATS and CK1-૪. With 320 its defined TEAD binding domain and WW domain, it could interact with transcription factor TEAD and other reported PPXY domain-containing proteins. 


\section{Structural features of YAP and TEAD interaction}

324 The Hippo effector protein YAP is known to elicit its biological function as transcription co-effector

325 by interacting with transcription factors. Presently, YAP is known to interact with TEAD, $\beta$-catenin,

326 SMAD, RUNX, p73 and ErbB4 for regulating their transcriptional responses as an activator or

327 repressor (Strano et al., 2001; Komuro et al., 2003; Zhao et al., 2008; Szeto et al., 2016; Passaniti et

328 al., 2017; Pan et al., 2018). Among these, YAP-TEAD interaction has been extensively studied and is

329 known to be important for regulating cell growth and size as well as tissue architecture (Totaro et al.,

330 2018). The interaction of YAP and TEAD was first shown to form through their specific interaction

331 domains in 2001 (Vassilev et al., 2001). The structural features of this interaction in humans were

332 first demonstrated in 2009 showing how the TEAD binding domain (TBD) in YAP (amino acids 53-

333 99) interacted with the YAP binding domain (YBD) in the TEAD (position: amino acids 209-426)

334 (Li et al., 2010). The YBD consists of $12 \beta$ strands which arrange themselves into two $\beta$ sheets in an

335 opposing fashion to form a $\beta$-sandwich fold. The four $\alpha$ helices from the YBD are arranged at the two

336 ends of the $\beta$-sandwich fold for stabilizing the structure. The study showed that TBD-YBD

337 interaction occurs over 3 interfaces. Each interface consisted of one of the following secondary

338 structure of the TBD- the $\beta 1$ strand, $\alpha 1$ helix or $\alpha 2$ helix responsible for interacting with the globular

339 YBD of the TEAD at the C-terminal. It was shown that the $\beta 1$ strand of TBD interacted with the $\beta 7$

340 strand of the YBD (interface 1), The $\alpha 1$ helix from TBD interacted with $\alpha 3$ and $\alpha 4$ helices of the

341 YBD (interface 2). The $\alpha 2$ of the TBD was bound to the YBD through its interaction with $\alpha 1$ and $\alpha 2$

342 helices (interface 3) (Li et al., 2010).

343 Amino acid sequence alignment of the predicted YBD (amino acids 240-251) and predicted TBD

344 (position: 1-58) of $\mathrm{Hvul}_{-}$YAP and $\mathrm{Hvul}$ TEAD respectively with Human YAP and TEAD revealed

$34571.2 \%$ sequence identity ( $82.9 \%$ sequence similarity) of YBD (Figure 2 A) and a $37.9 \%$ sequence

346 identity (56.9\% sequence similarity) of TBD (Figure 2 B) which indicates plausible structural

347 conservation and hence interacting capability of TBD with YBD. To confirm the same, the 3D

348 structure of the YBD and TBD of Hvul_YAP and Hvul_TEAD was modelled using MODELLER

349 software (Webb and Sali, 2016). The modelling was done based on the 4RE1 X-ray diffraction

350 structure deposited at Research Collaboratory for Structural Bioinformatics PDB (RCSB PDB-

351 https://www.rcsb.org/) which models the interaction of human homologs of TBD and YBD at a

352 resolution of $2.20 \AA$. The model generated from the Hydra homologs was superimposed on the

353 human YAP (hYAP) and hTEAD structure from 4RE1 and was found to highly structurally similar

354 (RMSD for $H v u l \_Y A P: h Y A P-0.338 \mathrm{~A}^{\circ}$ and for $H v u l$ TEAD:hTEAD- $0.310 \mathrm{~A}^{\circ}$ ) and indicated a 
conserved interaction capability of $H v u l \_Y A P$ and $H v u l \_T E A D$ (Figure $2 \mathrm{C}$ ). The modeled YBD-

356 TBD complex of Hydra clearly shows how three different regions- Region 1, Region 2 and Region 3 of TBD (purple) interacts with the globular YBD (green) by non-covalent bond interactions

358 (Supplementary Figure 3A). The Region 1 interface consisting of TBD $\beta 1$ (amino acids 10-17) and YBD $\beta 7$ (358-363) strands interact with seven hydrogen bonds in the human complex, forming an anti-parallel $\beta$ sheet (Li et al., 2010). In Hydra there are only six hydrogen bonds (green dotted lines) due to the presence of Gln18 in $\beta 1$ instead of Gly59 found in humans (Li et al., 2010), introducing a rotation in the preceding Arg which disables it from forming a hydrogen bond (Supplementary Figure $3 B$ ). The $2^{\text {nd }}$ interface (Region 2) has the $\alpha 1$ helix of the TBD (amino acids 20-32) fitting right into the binding groove of the YBD formed by the $\alpha 3$ and $\alpha 4$ helices of the YBD (amino acids 385-409) (Supplementary Figure 3C). Similar to humans; this region is mainly mediated by hydrophobic interactions with the $\alpha 1$ helix of the TBD having conserved LXXLF motif for hydrophobic groove binding (Li et al., 2010). This interaction mainly consists of Leu24, Leu27and Phe28 from TBD and lines) not found in humans may lead to a more stable interface. The $3^{\text {rd }}$ region $\left(3^{\text {rd }}\right.$ interface) consists of a twisted coil and $\alpha 2$ helix (amino acids 42-58) from the TBD interacting deeply with the pocket formed by the $\alpha 1$ helix, $\beta 4, \beta 11$ and $\beta 12$ helices of the YBD. This region was found to be indispensable for the YAP-TEAD complex formation in humans (Li et al., 2010). The region 3 in Hydra contains the hydrophobic side chains of the TBD - Phe44 (Met86 in humans), Leu49, Pro50 and Phe53 forming extensive van der Waals interactions with the YBD of TEAD at Glu280, Ala281, Ile282, Gln286, Ile287, Leu312, Leu316, Val431, His444 \& Phe446 (Supplementary Figure 3D). The interface is further strengthened by multiple hydrogen bonds (indicated in green dotted lines) Phe44 from TBD and Lys314, Glu408 and Phe446 from the YBD. While the hydrophobic interactions involving Pro56 and Pro57 from TBD with Trp316 and His444 respectively help to push the proline residues out of the hydrophobic pocket. One of the unique aspects that can be predicted from the model is that Hydra region 3 YAP-TEAD complex is able to form two salt bridges (orange dotted lines) - TBD_Arg47:YBD_Asp289:YBD_Asp289 and TBD_Lys48:YBD_Asp283:YBD_Asp451. The human complex only forms a salt bridge at TBD_Arg89:YBD_Asp249: YBD_Asp249. These observations indicate a more stable YAP-TEAD interaction in Hydra as compared to the humans. To shed more light on the same, the computationally calculated binding energy of the YAP-TEAD complex between the two organisms 
were compared using the web-based server PRODIGY (PROtein binDIng enerGY prediction) in

Protein-protein mode (Xue et al., 2016). The $\Delta \mathrm{G}$ of YAP-TEAD complex in humans is about -6.8 kcal mol${ }^{-1}$ while the complex in Hydra has a value of $-14.7 \mathrm{kcal} \mathrm{mol}^{-1}$. This large difference in the binding energy supports the possibility that the YAP-TEAD complex in Hydra is much more stable.

\section{Expression analysis of the Hippo pathway genes in Hydra}

393

394

395

396

397

398

399

400

401

402

403

404

405

406

407

408

409

410

411

412

413

414

415

416

417

\section{Hvul_yap expression in Hydra}

The expression pattern of Hvul_yap in Hydra polyp was studied by whole-mount in situ hybridization (WISH). The staining pattern observed from the whole polyp indicates low-level expression throughout the body with higher expression at the tentacle base and tip of the early stages of the developing new bud (Figure 3A). A closer look indicates that the expression is stronger in the endodermal cells as compared to the ectodermal cells (Figure 3B - E). yap expression in the early stages of bud development indicates its role in budding. Higher yap expression at the region of high mechanical stress such as the tentacle base, early budding tip and mature bud-parent polyp boundary indicates a probable ancient mechano-sensory role of YAP in Hydra. These polyps were cryosectioned to obtain a closer look at the types of cells expressing yap (Supplementary Figure 4). The images of these sections revealed cells in doublets, quadruplets and groups of cells among other stained cells indicating their interstitial stem cell origin, plausibly nematoblast and nests of nematoblasts.

\section{Expression pattern of $\mathrm{Hvul} \_$hpo, Hvul_mob and Hvul_savgenes}

An RNA WISH study of Hvul_hpo showed expression throughout the gastric region (Figure 4A). No expression was observed at the differentiated zones of hypostome, tentacle or basal disk which might indicate a role in stem-cell maintenance or differentiation but not in terminally differentiated cells. There is a slight reduction in expression at the budding zone and early buds which might indicate the antagonistic role of HPO towards YAP activity in areas of high mechanical stress as reported in other organisms. Hvul_hpo expression can also be seen at mature bud-parent polyp boundary indicating a fine-tuning of regulation of Hippo pathway-dependent during bud detachment. Hvul_mob expression showed a similar pattern to that of Hvul_yap with a distinct down-regulation at the basal disk region of both adult and budding Hydra (Figure 4B). Hvul_sav expression reflected the expression pattern of Hvul_hpo indicating a similar role. It can also be noted that there is marked reduction in expression at the budding region, early and late buds, unlike the Hvul_hpo (Figure 4C). 
418 A recent study reported high-throughput sequencing of the transcriptome of 24,985 single Hydra

419 cells using Drop-seq and identified the molecular signatures of various cell states and types (Siebert

420 et al., 2019). The differential expression of the Hippo pathway components and their pattern were

421 examined using the Single Cell Portal. The expression patterns of Hvul_yap, Hvul_tead, Hvul_hpo,

422 Hvul_lats, Hvul_sav\&Hvul_mob were queried. From the single-cell data, Hvul_yap expression was

423 found to be insignificantly dysregulated or differentially expressed between cell-types

424 (Supplementary Figure 5). Surprisingly, such a trend was commonly observed between all the other

425 Hippo pathway components namely, Hvul_tead, Hvul_hpo, Hvul_lats, Hvul_sav\&Hvul_mob. This

426 indicates a slight disparity with the WISH data. This could be due to lack of enough resolution from

427 the datasets used. The data showed here only represent a relative fold-change between the cells and

428 may indicate that the expression levels are relatively the same between the cells. The WISH data also

429 indicate that most of the cells express almost all types of Hippo pathway components, yet at the same

430 time, we see that they are excluded from some regions. These may be extremely stage-specific and

431 hence difficult to be picked up in sc-RNAseq of whole polyps. Nevertheless, the findings from

432 analyzing single-cell data argue in favor of the fact that the Hippo pathway components are essential

433 for cells and need to be expressed in almost all cell-types. Their activity might be regulated at the

434 protein level, and hence a protein-based analysis is essential to better understand the regulation of

435 Hippo pathway in Hydra.

437 Protein Expression analysis of the Hvul_YAP in Hydra

438 Region-specific and cell-type expression of $H_{v u l}$ YAP in $H y d r a$

439 The Clytia hemispherica specific Yorkie (CheYki) antibody was raised against a peptide from the

440 WW1 region of CheYorkie in rabbit (Coste et al., 2016). The CheYki peptide sequence was extracted

441 from the Marine Invertebrate Model Database (MARIMBA) and was used to align with Hvul_YAP

442 using CLUSTAL Omega. The full protein alignment showed just a $39.36 \%$ identity. However, a

443 peptide-specific (immunogen) alignment gave a $60 \%$ identity which raised the probability of cross

444 reactivity of this antibody against $\mathrm{Hvul}_{-}$YAP (Supplementary Figure 6 A). To test the same, an

445 immunofluorescence assay (IFA) was run using CheYki antibody or pre-immune serum. The IFA

446 yielded a robust signal for CheYki antibody as compared to the negative control (Supplementary

447 Figure 6 B). Examination of localization of YAP expressing cells revealed a pattern similar to what

448 we found in YAP ISH (Figure 5 A). The expression was seen more or less throughout the body. The 
449 base of the tentacle showed high expression similar to that seen in ISH but the number of YAP

450 expressing cells drops in hypostomal region and the inter-tentacle zone. Unlike the pattern of

451 transcripts seen in the ISH, the YAP expressing cells were depleted at the basal disk region.

452 The body column of Hydra is uniformly interspersed with YAP expressing cells (Figure 6). These

453 cells can be seen almost exclusively in groups (duplets, quadruplets or more). There were specific

454 patterns of these groups which looked similar to the ones seen in cryosections of ISH samples

455 (Supplementary Figure 4). The expression was clear for nuclearized YAP while the cytoplasmically

456 localized YAP were dispersed and difficult to observe. A careful analysis of cells expressing YAP

457 based on the staining intensity and intercellular distance, as seen in IFA indicates different subsets of

458 cells. Based on the YAP expression intensity, there seem to be cells exhibiting high expression (Blue

459 arrow), medium expression (yellow arrow) and low expression (green arrow). Based on the cellular

460 clustering, cell types can be divided into cells which are duplets or quadruplets (orange arrows)

461 which may be interstitial stem cell undergoing first and second mitotic division. There are also

462 clusters of cells which are arranged into a linear file whose identity is difficult to judge (red arrows).

463 Yellow arrows indicate clusters of cells which looks like part of a nest of nematoblasts. These nest

464 cells are typically arranged into 8-16 cell-clusters. As can be noticed here, these clusters are not

465 completely YAP expressing, and only a subset of these express YAP. This may indicate that these

466 cells are expressing only at certain stages of nematoblast differentiation. Such similar clusters can be

467 observed even in the high-level YAP expressing cells (blue arrows) indicating a yet different subset

468 of nematoblast cells. A different population of cells shows extra-nuclear staining (white arrow).

469 These stains might be non-specific since they are localized in cysts similar to that seen in

470 desmonemes and stenoteles. These results suggest that at least some of the YAP expressing cells

471 have interstitial cell origin. While inner hypostome (area immediate around the mouth) and the

472 tentacles are virtually devoid of YAP expressing cells, we find that there are a few non-clustered

473 YAP expressing cells at the region interstitial to the tentacle bases and the outer hypostome (Figure 5

474 and Supplementary Figure 7B). Such an expression pattern may indicate a role of YAP in tissue

475 compartment-boundary regulation for hypostomal and tentacle development and/or maintenance of

476 gene networks in Hydra. Such a role of Yki (YAP) has been recently proposed in Drosophila in wing

477 imaginal disc development by regulating the expression of Hox genes and Hedgehog signaling

478 (Bairzin et al., 2020). 


\section{YAP expressing cells are recruited to newly developing buds but are excluded from the}

\section{hypostomal region upon initiation of differentiation}

481 Cellular dynamics of YAP expressing cells during Hydra bud development was studied using immunofluorescence assay. Buds at different points of bud-development from early to late stages were observed (Stage 3, 4, 6 and 9). It was clear that the YAP expressing cells moved into the early bud with an expression pattern very similar to that found in the body column. Such pattern is persistent throughout the budding stages in the body column of the newly developed bud. The most interesting changes happening to the YAP expressing cells in a bud is at the hypostomal region. The YAP expressing cells near the distal bud tip were found to be non-clustered as compared to the rest of the lower bud region. At stage 3, the bud-tip where the head organizer has been set for establishing the new body axis for bud, YAP expressing cells seems to be depleted (Figure 7A). This pattern is even more conspicuous from stage 4 onwards (Figure 7B-D, Supplementary Figure 7A). From stage 9 onwards, the expression pattern similar to the adult Hydra is established where we see nonclustered YAP expressing cells seen sparsely at the boundaries between the hypostome and the tentacle base (Figure 7D, Supplementary Figure 7B). The appearance of non-clustered cells in these regions may indicate a different sub-type of YAP expressing cells having a role in head organizer maintenance in Hydra. Another interesting point to note is that YAP expressing cells are completely depleted at the basal disk (Figure 5), hypostome and tentacles. This observation may indicate an important antagonistic role of YAP signaling in tissues with terminally differentiated cells. The lack of YAP expressing cells even at the early developmental stages of tentacle development in a new bud (Supplementary Figure 7B) and at the Adult-bud boundary where the basal disk will form (Supplementary Figure 7C) further suggests the possibility of Hippo pathway in cell differentiation.

\section{YAP expressing cells are early responders to head amputation}

502 Immunostaining for YAP on decapitated polyps shed light on the participation of YAP expressing

503 cells during early regeneration (Figure 7). YAP expressing cells can be observed occupying the site

504 of injury within one hour of amputation. These cells increase in density as time progresses until 4

505 hours post-amputation (hpa). Since wound healing takes approximately 1-2 hpa, the YAP expressing

506 cells may migrate along with the epithelial cells during the wound-healing phase. An increase in

507 density might be caused by either further migration of the YAP expressing cells from the body

508 column or by division from the pre-existing cells at the site of injury. The interesting observation to

509 note is that the population of YAP expressing cells at the site of injury until 4 hpa is similar to the 
510 population seen in the body column of Hydra (green arrows). This changes after 8 hpa as the YAP

511 expressing cells at the distal-most region of the regenerating tip assumes a new cell-type

512 characteristic. These cells are non-clustered and look similar to the cells observed at the budding tip

513 and at the region around the boundaries of hypostome and tentacle base in adult Hydra. They can

514 now be seen as individual cells arranged arbitrarily at the tip. Since YAP is a known

515 mechanotransducer, these cells are either differentiated from the cells migrated from the body

516 column or are cells assuming a new phenotype in response to the mechanical change in the cellular

517 environment due to lack of ECM and physical disruption of cells (Shimizu et al., 2002). The

518 dynamics of YAP expressing cells in the regenerating tips indicate that they are recruited to the site

519 of injury early during the regeneration and are probably early responders of mechanical changes.

\section{Discussion}

522 Detailed characterization of the Hippo pathway and its components in pre-bilaterians has been

523 extremely sparse. There have been few studies reporting the presence of Hippo homologs in these

524 primitive organisms. Capsaspora owczarzaki, a single-celled eukaryote is the most primitive

525 organism predicted to have a complete set of functional core Hippo pathway homologs indicative of a

526 holozoan origin of the functional pathway (Sebé-Pedrós et al., 2012). Another study confirmed the

527 presence of Hippo pathway components in a Ctenophore species: Pleurobrachia pileus and a

528 Cnidarian species Clytia hemispherica (Coste et al., 2016). While this study reported an absence of

529 Yki in Ctenophores, it showed that the Yki in Clytia are conserved for the regulation of cell

530 proliferation and growth. In this study, we have for the first time identified and characterized a

531 complete set of core Hippo pathway components in Hydra vulgaris through bioinformatic analysis

532 and cloning. The current phylogenetic analysis is in congruence with previous report that

533 Nematostella vectensis homologue is more similar to complex vertebrates (Hilman and Gat, 2011). In

534 fact, all the Cnidarian homologues exhibit higher similarity with the chordate YAP sequences. This

535 suggests that YAP sequences evolved close to the emergence of the chordate homologs and might

536 exhibit similar properties observed in these organisms. Domain organization analysis indicates

537 divergence in the N-terminal homology domain of YAP (FAM181) in different lineages. This

538 suggests the clade specific role of the FAM181 region, probably in the interactions with TEAD like

539 or other proteins. This further indicates taxon-specific modification took place in the FAM181 region

540 and might play lineage specific functions. We show that the Hippo pathway components are more or 
541 less uniformly expressed throughout the polyp tissues barring a few regions in a gene-specific

542 manner like budding zone, early buds, extremities of the polyps such as tentacle tips or basal disks.

543 Considering the studies in bilaterians indicating that these components are all tightly controlled to

544 regulate the cell cycle and cell differentiation, it can be easily seen why these genes are expressed

545 uniformly in all tissues. Since the extremities of the polyps are terminally differentiated, they

546 probably do not need these genes for the functions mentioned above and are already set to perform its

547 designated functions without needing any change. The analysis of amino acid sequences of these

548 genes to predict the secondary structure and 3D tertiary protein models have also given us some

549 insightful results. Domain architecture of all the Hippo pathway proteins shows that their architecture

550 is well conserved in Cnidaria, which confirms an ancient establishment and evolution of the pathway

551 in the basal metazoans. The 3D modelling of YAP's TBD and TEAD's YBD in Hydra using the

552 published crystal structure of their Human homolog predicts a similar interaction capability of YAP

553 and TEAD in Hydra. Our analysis revealed that the YAP-TEAD complex is highly stable in Hydra.

554 This raises the possibility that the YAP-TEAD interaction was robust in primitive metazoans, and as

555 the signaling pathway evolved, the stability of the complex was presumably partially compromised to

556 accommodate the promiscuous nature of YAP in more complex organisms. This indirectly indicates

557 that the functions of the Hippo pathway or YAP signaling reported in bilaterians may have been

558 established as early as in Cnidarians and hence may have played in developing important

559 characteristics of multicellular organisms like cell-type divergence, body-axis development, germ-

560 layer differentiation etc.

561 In Clytia, it was found that Yki was nuclearized at the tentacle base where there are highly

562 proliferating cells, while they are inhibited in the tentacles where the cells are differentiated (Coste et

563 al., 2016). Using the antibodies used in the same study, we were able to study the protein-level

564 expression of YAP in Hydra. We find that even though Hvul_yap is expressed uniformly throughout

565 the polyp, only a few cells have Hvul_YAP in the "active form" (nuclearized). We find that these

566 nuclearized YAP are more or less uniformly spread throughout the polyp. YAP expression is almost

567 absent or not nuclearized in the terminally differentiated regions including the tentacles, hypostome

568 or basal disk. An interesting observation is the presence of YAP expressing cells at the tentacle base

569 forming a circle (Figure 5 and Supplementary Figure 7B). This can be considered homologous to the

570 expression pattern seen in Clytia which may be speculated as necessary for terminal differentiation of

571 cells while crossing the body column-tentacle boundary. Another possibility can be the mechanical

572 activation due to physical stress experienced at the tentacle base due to movement of tentacles or 
573 anatomical constraints. Most of these cells in the body column can be seen in groups or colonies.

574 Cellular features and arrangements of YAP positive cells are indicative of interstitial stem cell origin.

575 Cells like desmonemes and stenoteles are mechano-sensitive, and YAP may regulate their

576 development and function. Another interesting observation is the presence of a non-clustered group

577 of cells in the outer hypostomal region (Supplementary Figure 7B). Such an expression pattern raises

578 many interesting possibilities. It is reported that the ectodermal cells in the hypostome is maintained

579 separately from the gastric region (Dübel et al., 1987;Dübel, 1989). The inner hypostomal ring

580 consists exclusively of terminally differentiated cells (Dübel, 1989). The stationary region in the

581 hypostome (the outer hypostomal ring) contains a population of the ectodermal epithelial cells that

582 retains its proliferative potential which contribute exclusively to the cell-types in the entire

583 hypostomal region. Once the hypostome is specified, there are no contributions from the gastric

584 ectoderm towards hypostomal cells unless the hypostome is lost upon amputation. A unique

585 population of YAP expressing cells (non-clustered cells) in the outer hypostomal region and not at

586 the inner region may indicate the possibility of these cells being maintained in their undifferentiated

587 proliferative stem cell state by YAP for specific hypostomal functions. An identical population of

588 cells can be found at the regenerating tip (Figure 8-white arrows) and during early bud development.

589 These observations raise the possibility of these cells having a crucial role in establishing and

590 maintaining the head organizer. This regulation may well be mechanically activated upon amputation

591 or biochemically with pre-existing cues. The appearance of the non-clustered cells at the regenerating

592 tip at $8 \mathrm{hrs}$ is interesting since the expression of one of the variants of brachyury (HyBra2) coincides

593 with the same time point in the regenerating Hydra ((Bielen et al., 2007); Unni et al., unpublished

594 findings). The same study also shows early expression of Bra during bud formation. Interestingly, the

595 expression pattern of $\mathrm{HyBra}$ is exclusively at the hypostomal region encompassing both the outer and

596 inner hypostome (Technau and Bode, 1999; Bielen et al., 2007). Bra is known to be a direct

597 responder to consolidation Wnt/ $\beta$-catenin signaling (Yamaguchi et al., 1999). HyBra has also been

598 implicated in the establishment of the head organizer in Hydra (Technau and Bode, 1999). Hence,

599 this may mean that appearance of HyBra may coincide with the true setting up of the head organizer.

600 This raises the enticing prospect of YAP expressing cells at the outer hypostome region to restrict the

601 head organizer-related function of Brachyury to the inner hypostome ring by exerting its tissue

602 boundary regulation functions via the hedgehog pathway (Bairzin et al., 2020). Taken together with

603 the expression pattern of YAP in developing bud, adult polyp and the regenerating tip, consolidates

604 the possibility of YAP in the establishment and maintenance of the head organizer function in Hydra. 
605 This study shows that the Hippo pathway is an important signaling pathway capable of regulating the

606 cellular differentiation and tissue regeneration in Hydra. A more in-depth study of YAP signaling

607 under these contexts might reveal interesting insights into the evolution of the functions associated

608 with complex organisms. YAP can act as a mechanotransducer and has been shown to play a role in

609 regulating various morphogenetic and developmental functions. This aspect of YAP is only starting

610 to be fully understood and have been poorly studied in basal metazoans to understand its origins. A

611 detailed study in Hydra to understand the same will shed light on the fundamental aspects of how

612 tissue mechanics plays a role in regulating cell function.

\section{Conflict of Interest}

615 The authors declare that the research was conducted in the absence of any commercial or financial 616 relationships that could be construed as a potential conflict of interest.

\section{Author Contributions}

618 Conceptualization: M.K.U., P.C.R., S.G.; Methodology: M.K.U., P.C.R., S.G; Validation: M.K.U., 619 P.C.R., S.G; Formal analysis: M.K.U., P.C.R., S.G.; Investigation: M.K.U. \& P.C.R.;

620 Resources: S.G.; Writing - original draft: M.K.U., P.C.R., S.G.; Writing - review \& editing: M.K.U., 621 P.C.R., S.G.; Visualization: M.K.U., P.C.R., S.G.; Supervision: S.G.; Project administration: S.G.;

622 Funding acquisition: S.G.

\section{$623 \quad$ Funding}

624 This work was supported by the Centre of Excellence in Epigenetics program (BT/01/COE/09/07) of the 625 Department of Biotechnology, Government of India and the JC Bose National Fellowship from the 626 Science and Engineering Research Board (JCB/2019/000013) (S.G.). The authors acknowledge funding 627 from IISER Pune - intramural (S.G); Department of Biotechnology postdoctoral fellowship (P.C.R); and

628 Early Career Fellowship (IA/E/16/1/503057) (P.C.R.); fellowships from the University Grants

629 Commission (UGC) (M.U.); EMBO Short-term fellowship and Infosys Foundation for international 630 travel support (M.U.) 


\section{Acknowledgments}

634 Authors wish to thank Dr Michaël Manuel for providing the kind gift of CheYki antibody, and Dr Inna

635 Solomonov for useful comments on the manuscript and Dr Neeladri Sen for his help with protein 636 modelling. We thank Prof Irit Sagi to allow use of facilities in her lab to perform Hydra YAP budding 637 immunofluorescence assay and Mr Assaf Hanuna for maintaining Hydra culture at the Weizmann 638 Institute of Science. We would like to thank Dr Yoseph Addadi for assistance with imaging samples 639 using Andor Dragonfly Spinning Disc Microscope, Dr Rachel Paul for assistance with whole-mount RNA 640 in situ hybridization of $H v u l \_m o b$, and the IISER-Pune Microscopy facility.

\section{Data Availability Statement}

642 The datasets for this study can be found in the Supplementary Table 1. This includes the protein 643 sequences used for phylogenetic analysis, the mRNA sequences and protein sequences of the $H y d r a$ 644 Hippo pathway core homologs in separate tabs of the excel sheet.

\section{References}

646

647

648

649

650

651

652

653

654

655

656

657

658

659

660

661

662

663

664

665

666

667
Bairzin, J.C., Emmons-Bell, M., and Hariharan, I.K. (2020). The Hippo pathway coactivator Yorkie can reprogram cell fates and create compartment-boundary-like interactions at clone margins. Science advances 6, eabe8159.

Bielen, H., Oberleitner, S., Marcellini, S., Gee, L., Lemaire, P., Bode, H.R., Rupp, R., and Technau, U. (2007). Divergent functions of two ancient Hydra Brachyury paralogues suggest specific roles for their C-terminal domains in tissue fate induction. Development 134, 4187-4197.

Biovia, D.S. (2017). BIOVIA Discovery Studio 2017 R2: A comprehensive predictive science application for the Life Sciences. San Diego, CA, USA http://accelrys. com/products/collaborative-science/biovia-discovery-studio.

Boggiano, J.C., Vanderzalm, P.J., and Fehon, R.G. (2011). Tao-1 phosphorylates Hippo/MST kinases to regulate the Hippo-Salvador-Warts tumor suppressor pathway. Developmental cell $21,888-895$.

Brunet, T., and King, N. (2017). The origin of animal multicellularity and cell differentiation. Developmental cell 43, 124-140.

Butterfield, N.J. (2000). Bangiomorpha pubescens n. gen., n. sp.: implications for the evolution of sex, multicellularity, and the Mesoproterozoic/Neoproterozoic radiation of eukaryotes. Paleobiology 26, 386-404.

Capella-Gutiérrez, S., Silla-Martínez, J.M., and Gabaldón, T. (2009). trimAl: a tool for automated alignment trimming in large-scale phylogenetic analyses. Bioinformatics 25, 1972-1973.

Chapman, J.A., Kirkness, E.F., Simakov, O., Hampson, S.E., Mitros, T., Weinmaier, T., Rattei, T., Balasubramanian, P.G., Borman, J., and Busam, D. (2010). The dynamic genome of Hydra. Nature 464, 592-596. 
668

669

670

671

672

673

674

675

676

677

678

679

680

681

682

683

684

685

686

687

688

689

690

691

692

693

694

695

696

697

698

699

700

701

702

703

704

705

706

Chen, Y., Han, H., Seo, G., Vargas, R., Yang, B., Chuc, K., Zhao, H., and Wang, W. (2019). The Hippo pathway origin and its oncogenic alteration in evolution. bioRxiv, 837500.

Coste, A., Jager, M., Chambon, J.-P., and Manuel, M. (2016). Comparative study of Hippo pathway genes in cellular conveyor belts of a ctenophore and a cnidarian. EvoDevo 7, 4.

Dübel, S. (1989). Cell differentiation in the head of Hydra. Differentiation 41, 99-109.

Dübel, S., Hoffmeister, S.A., and Schaller, H.C. (1987). Differentiation pathways of ectodermal epithelial cells in hydra. Differentiation 35, 181-189.

Edgar, R.C. (2004). MUSCLE: multiple sequence alignment with high accuracy and high throughput. Nucleic acids research 32, 1792-1797.

Franzenburg, S., Fraune, S., Künzel, S., Baines, J.F., Domazet-Lošo, T., and Bosch, T.C. (2012). MyD88-deficient Hydra reveal an ancient function of TLR signaling in sensing bacterial colonizers. Proceedings of the National Academy of Sciences 109, 19374-19379.

Fulford, A., Tapon, N., and Ribeiro, P.S. (2018). Upstairs, downstairs: spatial regulation of Hippo signalling. Current opinion in cell biology 51, 22-32.

Gierer, A., Berking, S., Bode, H., David, C.N., Flick, K., Hansmann, G., Schaller, C.H., and Trenkner, E. (1972). "Regeneration of hydra from reaggregated cells", in: Nature/New Biology.).

Glantschnig, H., Rodan, G.A., and Reszka, A.A. (2002). Mapping of MST1 kinase sites of phosphorylation: activation and autophosphorylation. Journal of Biological Chemistry 277, 42987-42996.

Guindon, S., and Gascuel, O. (2003). A simple, fast, and accurate algorithm to estimate large phylogenies by maximum likelihood. Systematic biology 52, 696-704.

Hergovich, A., Schmitz, D., and Hemmings, B.A. (2006). The human tumour suppressor LATS1 is activated by human MOB1 at the membrane. Biochemical and biophysical research communications 345, 50-58.

Hilman, D., and Gat, U. (2011). The evolutionary history of YAP and the hippo/YAP pathway. Molecular biology and evolution 28, 2403-2417.

Hobmayer, B., Rentzsch, F., Kuhn, K., Happel, C.M., Von Laue, C.C., Snyder, P., Rothbächer, U., and Holstein, T.W. (2000). WNT signalling molecules act in axis formation in the diploblastic metazoan Hydra. Nature 407, 186.

Horibata, Y., Sakaguchi, K., Okino, N., Iida, H., Inagaki, M., Fujisawa, T., Hama, Y., and Ito, M. (2004). Unique catabolic pathway of glycosphingolipids in a hydrozoan, Hydra magnipapillata, involving endoglycoceramidase. Journal of Biological Chemistry 279, 3337933389.

Huang, J., Wu, S., Barrera, J., Matthews, K., and Pan, D. (2005). The Hippo signaling pathway coordinately regulates cell proliferation and apoptosis by inactivating Yorkie, the Drosophila Homolog of YAP. Cell 122, 421-434.

Jones, D.T., Taylor, W.R., and Thornton, J.M. (1992). The rapid generation of mutation data matrices from protein sequences. Bioinformatics 8, 275-282.

Khalturin, K., Billas, I.M., Chebaro, Y., Reitzel, A.M., Tarrant, A.M., Laudet, V., and Markov, G.V. (2018). NR3E receptors in cnidarians: A new family of steroid receptor relatives extends the 
possible mechanisms for ligand binding. The Journal of steroid biochemistry and molecular biology 184, 11-19.

Komuro, A., Nagai, M., Navin, N.E., and Sudol, M. (2003). WW domain-containing protein YAP associates with ErbB-4 and acts as a co-transcriptional activator for the carboxyl-terminal fragment of ErbB-4 that translocates to the nucleus. Journal of Biological Chemistry 278, 33334-33341.

Krishnapati, L.-S., and Ghaskadbi, S. (2014). Identification and characterization of VEGF and FGF from Hydra. International Journal of Developmental Biology 57, 897-906.

Lemoine, F., Correia, D., Lefort, V., Doppelt-Azeroual, O., Mareuil, F., Cohen-Boulakia, S., and Gascuel, O. (2019). NGPhylogeny. fr: new generation phylogenetic services for nonspecialists. Nucleic Acids Research 47, W260-W265.

Lemoine, F., Entfellner, J.-B.D., Wilkinson, E., Correia, D., Felipe, M.D., De Oliveira, T., and Gascuel, O. (2018). Renewing Felsenstein's phylogenetic bootstrap in the era of big data. Nature 556, 452-456.

Letunic, I., and Bork, P. (2019). Interactive Tree Of Life (iTOL) v4: recent updates and new developments. Nucleic acids research 47, W256-W259.

Letunic, I., Goodstadt, L., Dickens, N.J., Doerks, T., Schultz, J., Mott, R., Ciccarelli, F., Copley, R.R., Ponting, C.P., and Bork, P. (2002). Recent improvements to the SMART domain-based sequence annotation resource. Nucleic acids research 30, 242-244.

Li, Z., Zhao, B., Wang, P., Chen, F., Dong, Z., Yang, H., Guan, K.-L., and Xu, Y. (2010). Structural insights into the YAP and TEAD complex. Genes \& development 24, 235-240.

Madden, T. (2013). "The BLAST sequence analysis tool," in The NCBI Handbook [Internet]. 2nd edition. National Center for Biotechnology Information (US)).

Martinez, D.E., Dirksen, M.-L., Bode, P.M., Jamrich, M., Steele, R.E., and Bode, H.R. (1997). Budhead, a fork head/HNF-3 homologue, is expressed during axis formation and head specification in hydra. Developmental biology 192, 523-536.

Mistry, J., Finn, R.D., Eddy, S.R., Bateman, A., and Punta, M. (2013). Challenges in homology search: HMMER3 and convergent evolution of coiled-coil regions. Nucleic acids research 41, e121-e121.

Moore, A.D., Held, A., Terrapon, N., Weiner 3rd, J., and Bornberg-Bauer, E. (2014). DoMosaics: software for domain arrangement visualization and domain-centric analysis of proteins. Bioinformatics 30, 282-283.

Münder, S., Tischer, S., Grundhuber, M., Büchels, N., Bruckmeier, N., Eckert, S., Seefeldt, C.A., Prexl, A., Käsbauer, T., and Böttger, A. (2013). Notch-signalling is required for head regeneration and tentacle patterning in Hydra. Developmental biology 383, 146-157.

Ni, L., Zheng, Y., Hara, M., Pan, D., and Luo, X. (2015). Structural basis for Mob1-dependent activation of the core Mst-Lats kinase cascade in Hippo signaling. Genes \& development 29, 1416-1431.

Otto, J.J., and Campbell, R.D. (1977). Budding in Hydra attenuata: bud stages and fate map. Journal of Experimental Zoology 200, 417-428. 
Pan, J.-X., Xiong, L., Zhao, K., Zeng, P., Wang, B., Tang, F.-L., Sun, D., Guo, H.-H., Yang, X., and Cui, S. (2018). YAP promotes osteogenesis and suppresses adipogenic differentiation by regulating $\beta$-catenin signaling. Bone research $6,1-12$.

Passaniti, A., Brusgard, J.L., Qiao, Y., Sudol, M., and Finch-Edmondson, M. (2017). "Roles of RUNX in Hippo pathway signaling," in RUNX Proteins in Development and Cancer. Springer), 435-448.

Perrimon, N., Pitsouli, C., and Shilo, B.-Z. (2012). Signaling mechanisms controlling cell fate and embryonic patterning. Cold Spring Harbor perspectives in biology 4, a005975.

Pettersen, E.F., Goddard, T.D., Huang, C.C., Couch, G.S., Greenblatt, D.M., Meng, E.C., and Ferrin, T.E. (2004). UCSF Chimera - a visualization system for exploratory research and analysis. Journal of computational chemistry 25, 1605-1612.

Philipp, I., Holstein, T.W., and Hobmayer, B. (2005). HvJNK, a Hydra member of the c-Jun NH2terminal kinase gene family, is expressed during nematocyte differentiation. Gene expression patterns 5, 397-402.

Potter, S.C., Luciani, A., Eddy, S.R., Park, Y., Lopez, R., and Finn, R.D. (2018). HMMER web server: 2018 update. Nucleic acids research 46, W200-W204.

Praskova, M., Khoklatchev, A., Ortiz-Vega, S., and Avruch, J. (2004). Regulation of the MST1 kinase by autophosphorylation, by the growth inhibitory proteins, RASSF1 and NORE1, and by Ras. Biochemical Journal 381, 453-462.

Price, M.N., Dehal, P.S., and Arkin, A.P. (2010). FastTree 2-approximately maximum-likelihood trees for large alignments. PloS one 5, e9490.

Reddy, P.C., Barve, A., and Ghaskadbi, S. (2011). Description and phylogenetic characterization of common hydra from India. Current Science 101, 736-738.

Reddy, P.C., Gungi, A., Ubhe, S., Pradhan, S.J., Kolte, A., and Galande, S. (2019a). Molecular signature of an ancient organizer regulated by Wnt/ $\beta$-catenin signalling during primary body axis patterning in Hydra. Communications Biology 2, 1-11.

Reddy, P.C., Gungi, A., and Unni, M. (2019b). "Cellular and Molecular Mechanisms of Hydra Regeneration," in Evo-Devo: Non-model Species in Cell and Developmental Biology. Springer), 259-290.

Reinhardt, B., Broun, M., Blitz, I.L., and Bode, H.R. (2004). HyBMP5-8b, a BMP5-8 orthologue, acts during axial patterning and tentacle formation in hydra. Developmental biology 267, 4359.

Ren, J., Wen, L., Gao, X., Jin, C., Xue, Y., and Yao, X. (2009). DOG 1.0: illustrator of protein domain structures. Cell research 19, 271.

Rentzsch, F., Guder, C., Vocke, D., Hobmayer, B., and Holstein, T.W. (2007). An ancient chordinlike gene in organizer formation of Hydra. Proceedings of the National Academy of Sciences 104, 3249-3254.

Schenkelaars, Q., Tomczyk, S., Wenger, Y., Ekundayo, K., Girard, V., Buzgariu, W., Austad, S., and Galliot, B. (2018). "Hydra, a model system for deciphering the mechanisms of aging and resistance to aging," in Conn's Handbook of Models for Human Aging. Elsevier), 507-520.

Sebé-Pedrós, A., Zheng, Y., Ruiz-Trillo, I., and Pan, D. (2012). Premetazoan origin of the hippo signaling pathway. Cell reports 1, 13-20. 
Shimizu, H., Zhang, X., Zhang, J., Leontovich, A., Fei, K., Yan, L., and Sarras, M.P. (2002). Epithelial morphogenesis in hydra requires de novo expression of extracellular matrix components and matrix metalloproteinases. Development 129, 1521-1532.

Siebert, S., Farrell, J.A., Cazet, J.F., Abeykoon, Y., Primack, A.S., Schnitzler, C.E., and Juliano, C.E. (2019). Stem cell differentiation trajectories in Hydra resolved at single-cell resolution. Science 365, eaav9314.

Sprinzak, D., Lakhanpal, A., Lebon, L., Santat, L.A., Fontes, M.E., Anderson, G.A., Garcia-Ojalvo, J., and Elowitz, M.B. (2010). Cis-interactions between Notch and Delta generate mutually exclusive signalling states. Nature 465, 86.

Strano, S., Munarriz, E., Rossi, M., Castagnoli, L., Shaul, Y., Sacchi, A., Oren, M., Sudol, M., Cesareni, G., and Blandino, G. (2001). Physical interaction with Yes-associated protein enhances p73 transcriptional activity. Journal of Biological Chemistry 276, 15164-15173.

Szeto, S.G., Narimatsu, M., Lu, M., He, X., Sidiqi, A.M., Tolosa, M.F., Chan, L., De Freitas, K., Bialik, J.F., and Majumder, S. (2016). YAP/TAZ are mechanoregulators of TGF- $\beta$-Smad signaling and renal fibrogenesis. Journal of the American Society of Nephrology 27, 31173128 .

Takaku, Y., Hwang, J.S., Wolf, A., Böttger, A., Shimizu, H., David, C.N., and Gojobori, T. (2014). Innexin gap junctions in nerve cells coordinate spontaneous contractile behavior in Hydra polyps. Scientific reports $4,3573$.

Tamura, K., Stecher, G., Peterson, D., Filipski, A., and Kumar, S. (2013). MEGA6: molecular evolutionary genetics analysis version 6.0. Molecular biology and evolution 30, 2725-2729.

Technau, U., and Bode, H.R. (1999). HyBra1, a Brachyury homologue, acts during head formation in Hydra. Development 126, 999-1010.

Tischer, S., Reineck, M., Söding, J., Münder, S., and Böttger, A. (2013). Eph receptors and ephrin class B ligands are expressed at tissue boundaries in Hydra vulgaris. International Journal of Developmental Biology 57, 759-765.

Totaro, A., Panciera, T., and Piccolo, S. (2018). YAP/TAZ upstream signals and downstream responses. Nature cell biology 20, 888-899.

Tweedt, S.M., and Erwin, D.H. (2015). "Origin of metazoan developmental toolkits and their expression in the fossil record," in Evolutionary transitions to multicellular life. Springer), 47-77.

Vassilev, A., Kaneko, K.J., Shu, H., Zhao, Y., and Depamphilis, M.L. (2001). TEAD/TEF transcription factors utilize the activation domain of YAP65, a Src/Yes-associated protein localized in the cytoplasm. Genes \& development 15, 1229-1241.

Watanabe, H., Schmidt, H.A., Kuhn, A., Höger, S.K., Kocagöz, Y., Laumann-Lipp, N., Özbek, S., and Holstein, T.W. (2014). Nodal signalling determines biradial asymmetry in Hydra. Nature $515,112$.

Waterhouse, A.M., Procter, J.B., Martin, D.M., Clamp, M., and Barton, G.J. (2009). Jalview Version 2 -a multiple sequence alignment editor and analysis workbench. Bioinformatics 25, 11891191.

Webb, B., and Sali, A. (2016). Comparative protein structure modeling using MODELLER. Current protocols in bioinformatics 54, 5.6. 1-5.6. 37. 
833

834

835

836

837

838

839

840

841

842

843

844

845

846

847

848

849

850

851

852

853

854

855

856

857

858

859

860

861

862

863

864

865

866

867

868

869

Wenger, Y., Buzgariu, W., Reiter, S., and Galliot, B. (Year). "Injury-induced immune responses in Hydra", in: Seminars in immunology: Elsevier), 277-294.

Xu, T., Wang, W., Zhang, S., Stewart, R.A., and Yu, W. (1995). Identifying tumor suppressors in genetic mosaics: the Drosophila lats gene encodes a putative protein kinase. Development 121, 1053-1063.

Xue, L.C., Rodrigues, J.P., Kastritis, P.L., Bonvin, A.M., and Vangone, A. (2016). PRODIGY: a web server for predicting the binding affinity of protein-protein complexes. Bioinformatics 32, 3676-3678.

Xue, Y., Liu, Z., Cao, J., Ma, Q., Gao, X., Wang, Q., Jin, C., Zhou, Y., Wen, L., and Ren, J. (2010). GPS 2.1: enhanced prediction of kinase-specific phosphorylation sites with an algorithm of motif length selection. Protein Engineering, Design \& Selection 24, 255-260.

Yamaguchi, T.P., Takada, S., Yoshikawa, Y., Wu, N., and Mcmahon, A.P. (1999). T (Brachyury) is a direct target of Wnt3a during paraxial mesoderm specification. Genes \& development 13, 3185-3190.

Yin, F., Yu, J., Zheng, Y., Chen, Q., Zhang, N., and Pan, D. (2013). Spatial organization of Hippo signaling at the plasma membrane mediated by the tumor suppressor Merlin/NF2. Cell 154, 1342-1355.

Zhao, B., Li, L., Tumaneng, K., Wang, C.-Y., and Guan, K.-L. (2010). A coordinated phosphorylation by Lats and CK1 regulates YAP stability through SCF $\beta$-TRCP. Genes \& development 24, 72-85.

Zhao, B., Ye, X., Yu, J., Li, L., Li, W., Li, S., Yu, J., Lin, J.D., Wang, C.-Y., and Chinnaiyan, A.M. (2008). TEAD mediates YAP-dependent gene induction and growth control. Genes \& development 22, 1962-1971.

Zhou, Z., Hu, T., Xu, Z., Lin, Z., Zhang, Z., Feng, T., Zhu, L., Rong, Y., Shen, H., and Luk, J.M. (2015). Targeting Hippo pathway by specific interruption of YAP-TEAD interaction using cyclic YAP-like peptides. The FASEB Journal 29, 724-732.

\section{Figure Legends:}

Figure 1: Identification of Hippo pathway homologs in Hydra. A. Domain architecture of the homologs as visualized using DOG 2.0. B. Depicts the percent identity of Hydra homolog with Drosophila and Human. C. Phylogenetic tree and domain organization of YAP homologs across the animal phyla. The phylogenetic analysis was carried out on NGphylogeny.fr webserver and the tree was generated using FastTree 2 method. Here, the phylogenetic tree was rooted at Amphimedon queenslandica YAP-like sequence (AMPQU). Domain organization analysis was carried out using DoMosaics software. Branch support values are displayed at the branching points. Different phyla are highlighted with distinct colours. Hydra YAP homologue (HVUL YAP) is highlighted in red colour 

the Supplementary Table 1.

872 Figure 2: YAP-TEAD interaction domain is structurally conserved in Hydra. A. Sequence

873 alignment of human and Hydra TEAD YAP-binding domains (YBD) showing 71.2\% sequence

874 identity. B. Sequence alignment of human and Hydra YAP TEAD-binding domains (TBD) showing $87537.9 \%$ sequence identity. The alignment consensus shows conserved amino acid residues at a given

876 position. If the there is no conservation, the position is labelled as X. Colour code: amino acid residues with positive charge- red, negative charge-blue and neutral-green. C. Structural superposition of predicted $H v u l \_$TEAD YBD and Hvul_YAP TBD with YBD and TBD complex in 879 Human (PDB:4RE1) showing highly conserved $\beta$ - strands and $\alpha$ - helices structural placement.

880 Important $\alpha$-helices and $\beta$-strands are indicated with their number identification which are involved 881 in the interaction of YBD and TBD. Colour code: Red- Hvul_YAP TBD, Blue- HVUL_TEAD YBD, 882 Green- human YAP TBD (PDB ID-4RE1), Purple- human TEAD YBD (PDB ID-4RE1). D)

883 Interaction of YBD (green color) with the TBD (purple color) in Hydra modelled using 4RE1

884 structure showing how the globular YBD (depicted in surface features) is bound by TBD (depicted as 885 ribbon) through interactions at three different regions -region 1, region 2, and region 3 . The amino acid side chains from TBD are represented as sticks for understanding their role in the interaction.

Figure 3: Hvul_yap expression analysis in Hydra. Whole-mount in situ hybridization of Hvul_yap

888 expression at A. Region across the polyp (inset shows polyp probed with a sense RNA probe). B.

889 Head, C. basal disk, D. mid-stage bud, E. early bud/ late bud foot. The scale bar is $500 \mu \mathrm{m}$ long.

890 Figure 4: Hvul_hpo, Hvul_mob and Hvul_sav expression analysis in Hydra. Whole mount in situ 891 hybridization of A. Hvul_hpo, B. Hvul_mob and C. Hvul_sav expression for the whole polyp. The 892 insets on the right indicate negative controls probed with sense RNA probe. The scale bar is $500 \mu \mathrm{m}$ 893 long.

894 Figure 5: Expression of $\boldsymbol{H v u l}$ Y YAP in Hydra. Immunofluorescence assay of Hvul_YAP performed 895 using anti-CheYki antibody showing localization of YAP positive cells at various locations in an 896 adult polyp. The red fluorescent dye shows Alexa 594 staining of YAP and the blue dye shows DAPI 897 staining of nucleus. The hypostomal region is indicated by a green box, the tentacle base is indicated 898 by an orange box. The body column is indicated by a blue box and basal disc area is indicated by a 899 red box. 
900 Figure 6: Types of $\mathbf{H v u l}$ Y YAP expressing cells in Hydra. Immunofluorescence assay of Hvul_YAP

901 performed using anti-CheYki antibody on macerated cells at 60X. A. This panel shows cell types

902 based on signal intensity or YAP expression level in cells. Blue arrow represents cells with high YAP

903 expression, yellow arrow represents cells with medium YAP expression, cells with a green arrow

904 represents low YAP expression. White arrow indicates extra-nuclear staining in nematocysts. B. This

905 panel depicts cell types based on the cellular arrangement. Orange arrows represent cells with duplet

906 or quadruplet arrangement and red arrow represents cells arranged linearly. Red: YAP \& Blue:

907 Nucleus (Magenta indicates merged image). Immunofluorescence assay using the anti- Hvul_YAP

908 antibody of macerated cells at 60X. The red fluorescent dye shows Alexa 594 staining of YAP and

909 the blue dye shows DAPI staining of nucleus. (Scale bar: $20 \mu \mathrm{m}$ )

910 Figure 7: YAP positive cells are recruited early to the bud tip and are excluded from the region

911 which are terminally differentiated in the late stages of bud development. Immunofluorescence

912 assay of $\mathrm{Hvul}$ YYAP performed using anti-CheYki antibody for different budding stages (represented

913 by two polyps for depicting each stage) of Hydra showing recruitment of YAP positive cells to the

914 budding tip. A. Stage 3 shows early recruitment of YAP positive cells to the emerging bud with non-

915 clustered cells at the distal tip with slight depletion at tip of the bud. B. at Stage 4, depletion of the

916 YAP expressing is more prominent which gets further exaggerated at C. Stage 6 and D. Stage 9. Red:

917 YAP \& Blue: DAPI. (Scale bar $=50 \mu \mathrm{m})$

918 Figure 8: YAP positive cells are recruited early to the regenerating tip in $\boldsymbol{H y d r a}$.

919 Immunofluorescence assay of $\mathrm{Hvul}_{-}$YAP performed anti-CheYki antibody for head regenerating

920 Hydra showing recruitment of YAP positive cells to the regenerating tip. The density of YAP

921 positive cells can be seen increasing at the site of injury from $1 \mathrm{hr}$ post-amputation ( $1 \mathrm{hpa})$. White

922 arrows indicated in the zoomed-in image shows the generation of a new type of YAP expressing cells

923 at the regenerating tip by $8 \mathrm{hpa}$ as compared to cell population seen away from the tip or previous

924 time points (green arrows) Red: YAP \& Green: Actin. $($ Scale bar $=50 \mu \mathrm{m})$ 


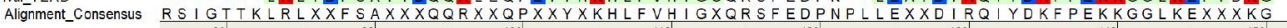

hTEAD

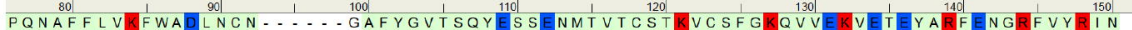
PQHIFFLVKFWADNTSIPDDAG S SYGVSTTYESSENMTVTCSTKVCSFGKQVVEKVETEYPRYENGRFVYRIO

Hvul_TEAD

Alignment_Consensus

hTEAD

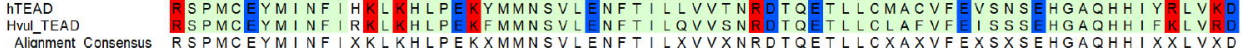
PQXXFFL VKFWADLNXXIPDDAGGXFYGVXXXYESSENMTVTCSTKVCSFGKQVVEKVETEYXRXENGRFVYRIX

TEAD: Identity: $71.2 \%$, Similarity: $82.9 \%$ (Region- YAP binding domain : 240-451)

B

hYAP

Hvul_YAP

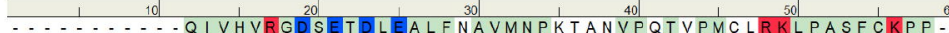

MDMNSTQRQGNFVLHVRQDSDTDLEQLFKNSVSTNKD-IPRSKPFRDRKLPASFFRPPP

Alignment_Consensus MDMNSTQRQGNXXXHVRXDSXTDLEXLFXXXXXXXXXNXPXXXPXXXRKLPASFXXPPP

YAP: Identity: 37.9\%, Similarity: 56.9\% (Region- TEAD binding domain : 1-58)

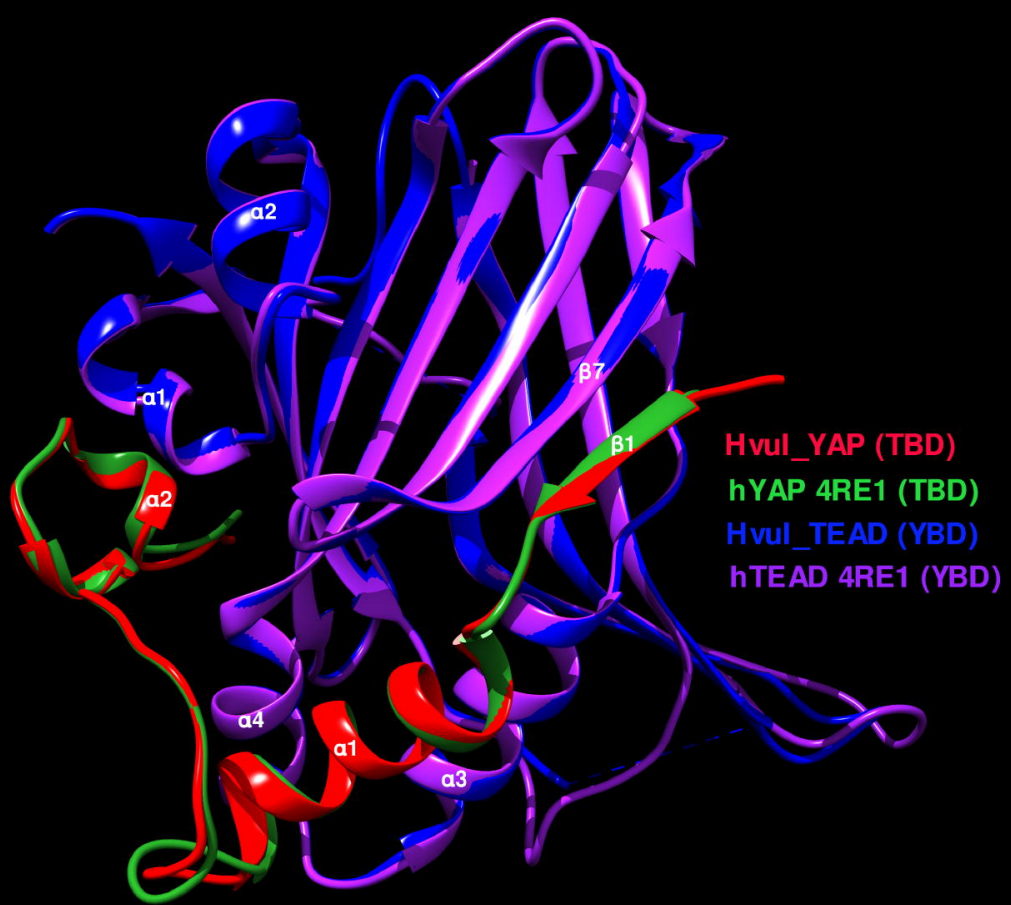



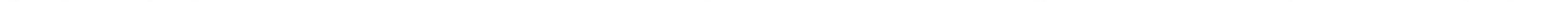


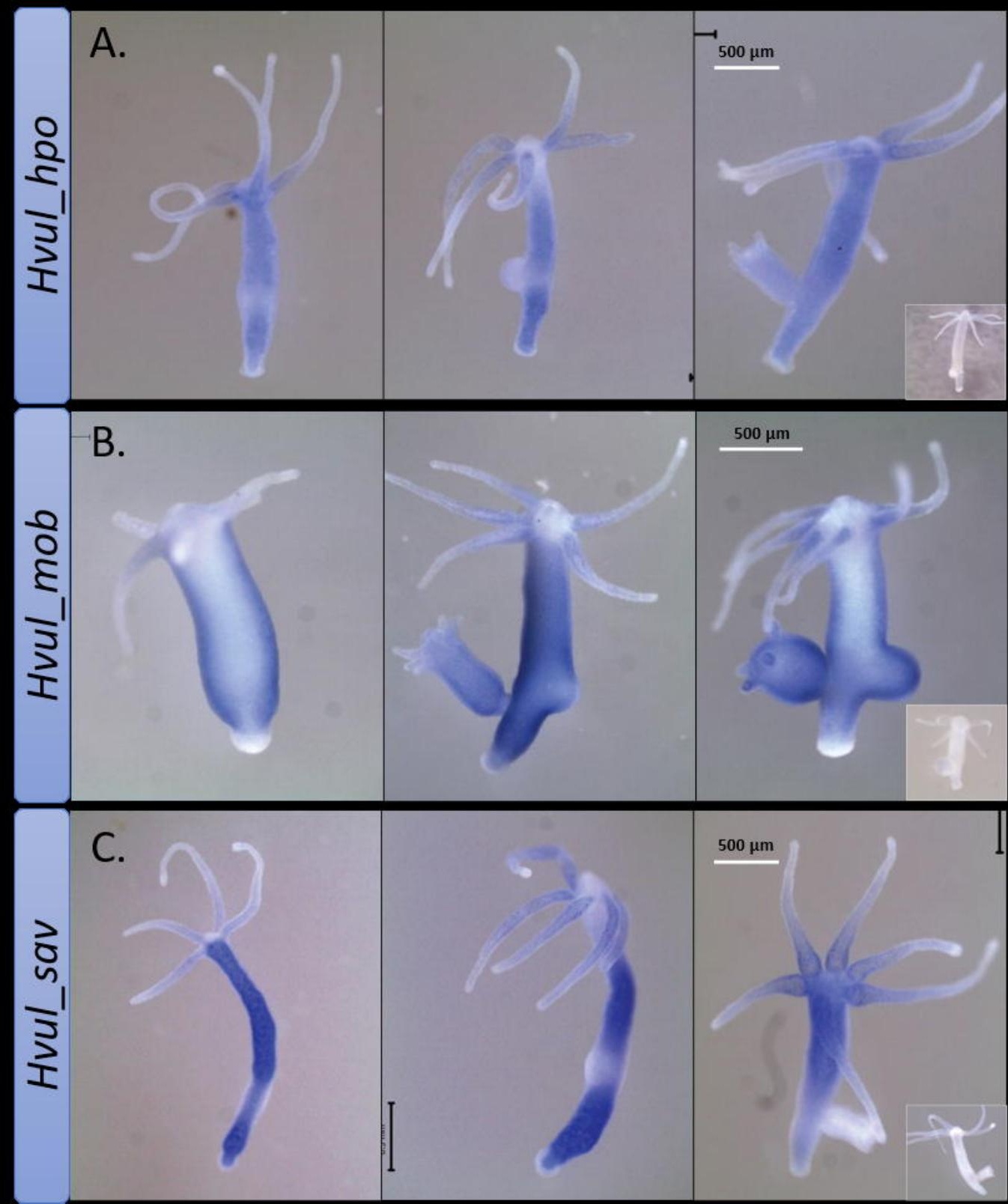



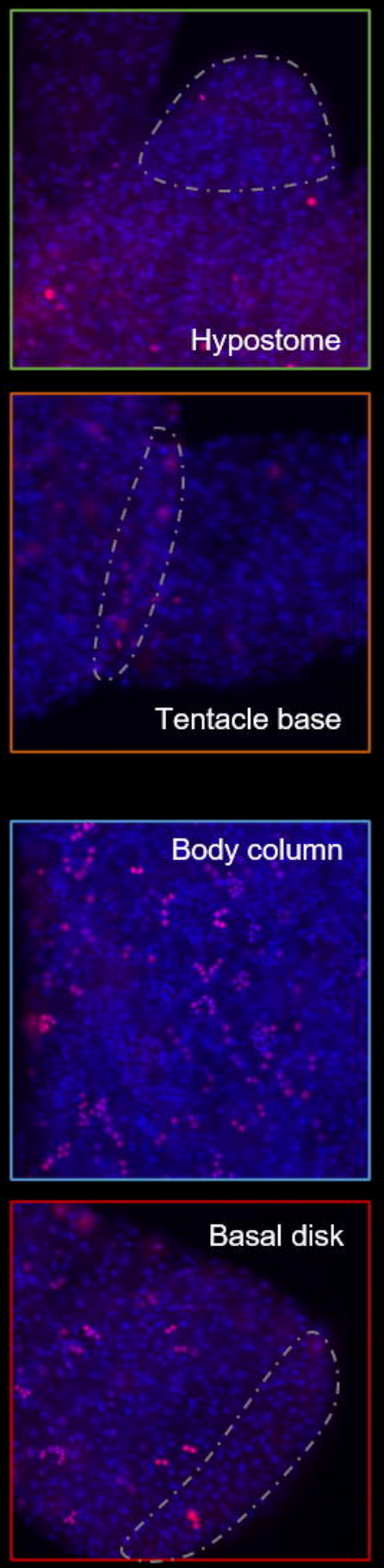
A.

B.

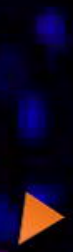

$\nabla$

D

$\Delta$

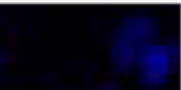

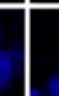

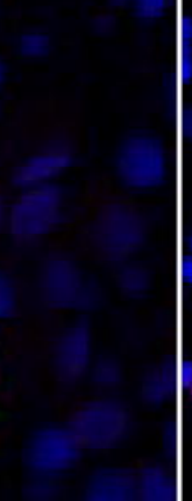




\section{A}

C

D

$\overline{50 \mu \mathrm{m}}$

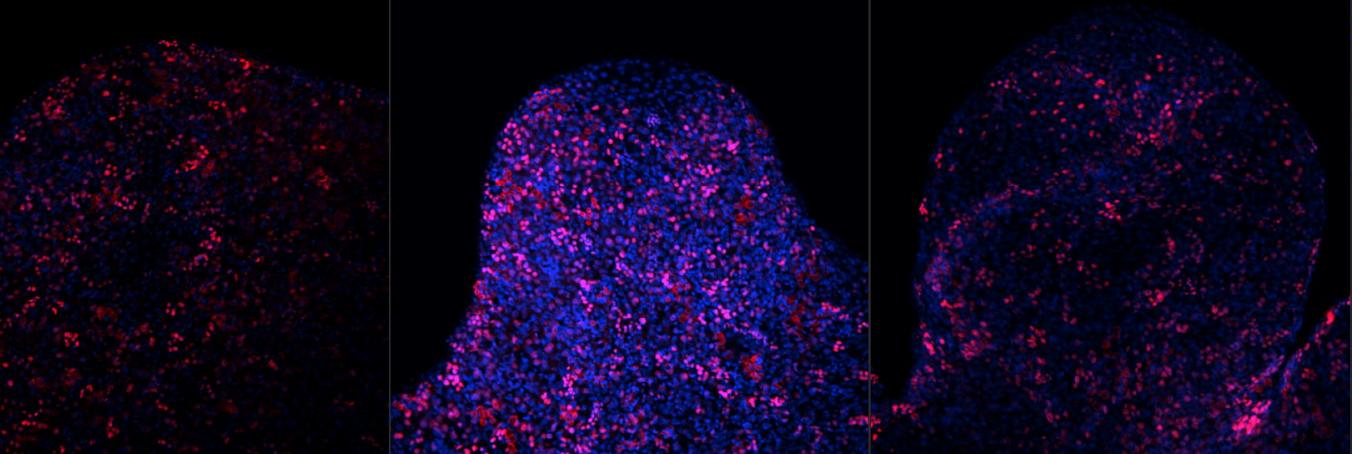

Stage 3

Stage 4

Stage 6

Stage 9 
